\title{
Salicylate Protects Hearing and Kidney Function from Cisplatin Toxicity without Compromising its Oncolytic Action
}

\author{
Geming Li, Su-Hua Sha, Elena Zotova, Joseph Arezzo, Thomas Van De Water, and \\ Jochen Schacht
}

Departments of Otolaryngology (GL) and Neuroscience (EZ, JA), Albert Einstein College of Medicine, Bronx, New York; Kresge Hearing Research Institute (S-HS, JS), University of Michigan, Ann Arbor, Michigan; and Department of Otolaryngology (TV), University of Miami, School of Medicine, Miami, Florida

\begin{abstract}
SUMMARY: Salicylate has recently been demonstrated to protect against the auditory and vestibular side effects of aminoglycoside antibiotics. Similarities in the toxic mechanisms suggest salicylate as a treatment strategy to prevent the ototoxic side effects of cisplatin (CDDP). We first tested protection of the inner ear in Wistar rats receiving a single infusion of $16 \mathrm{mg}$ CDDP/ $\mathrm{kg}$ body weight with or without treatment with $100 \mathrm{mg} / \mathrm{kg}$ salicylate (bid) for 5 days beginning one day before the CDDP infusion. Cisplatin induced a threshold shift of more than $30 \mathrm{~dB}$ (at $14 \mathrm{kHz}$; measured by auditory evoked brain stem response) that was significantly reduced by salicylate. We then examined the protective potential of salicylate on the cochlea, peripheral nerves, and kidney in a rat model of breast cancer-Fisher344 rats implanted with highly metastatic MTLn3 breast cancer cells. Animals received $3 \times 5 \mathrm{mg} \mathrm{CDDP} / \mathrm{kg}$ (given every third day), and salicylate was administered at $100 \mathrm{mg} / \mathrm{kg}$ (bid) from 2 days before to 3 days after CDDP treatment. Salicylate significantly attenuated the CDDP-induced threshold shift from approximately $20 \mathrm{~dB}$ (at 16 and $24 \mathrm{kHz}$ ) to approximately $5 \mathrm{~dB}$, and drastically reduced the loss of cochlear outer hair cells. Likewise, salicylate protected kidney function (measured as plasma blood urea nitrogen and creatinine levels) from CDDP toxicity. Protection of nerve conduction velocities of both sensory and motor nerves was minimal. The chemotherapeutic efficacy of CDDP on suppression of tumor mass and cancer cell metastasis remained unaffected by salicylate. The results suggest that administration of salicylate may become the basis of an effective therapeutic intervention against the ototoxic and nephrotoxic side effects associated with CDDP chemotherapy. (Lab Invest 2002, 82:585-596).
\end{abstract}

Cisplatin (cis-diamminedichloroplatinum [II];

$(\mathrm{CDDP})$ is a widely used chemotherapeutic agent in the treatment of a broad spectrum of tumors (Fram, 1992), but its severe side effects can limit its usage. These side effects include cumulative nephrotoxicity (Safinstein et al, 1986), neurotoxicity, ototoxicity (loss of hearing or balance or both), gastrointestinal toxicity, and myelosuppression (Blakley et al, 1994; Waters et al, 1991). In rats, CDDP exerts its nephrotoxic side effects mainly in the S3 segment of the proximal tubule of the kidney, resulting in necrotic lesions (Doyban et al, 1980); in humans, additional damage has been reported in the distal part of the tubules of the kidney (Gonzalez-Vitale et al, 1977). Manifestations of ototoxicity in both experimental animals and humans are a sensorineural hearing loss beginning at the high frequencies and based on the destruction of

Received January 16, 2002.

This research was supported by research grants DC-03685 from the National Institute on Deafness and Other Communication Disorders, $\mathrm{Na}$ tional Institutes of Health (to JS); and from the Singer Foundation of Beth Israel Medical Center (to TV).

Address reprint requests to: Dr. Thomas $R$. Van De Water, Department of Otolaryngology, University of Miami Ear Institute, 1600 NW 10th Ave., RMSB 3160,Miami,FL 33136.E-mail: tvandewater@med.miami.edu cochlear sensory cells (inner and outer hair cells), degeneration of the stria vascularis, and a significant decrease in spiral ganglion cells (Rybak et al, 1995). Because mammalian hair cells do not regenerate, the hearing loss is permanent. The incidence of auditory hair cell damage and hearing loss in patients undergoing CDDP chemotherapy may be as high as $90 \%$ (Schweitzer, 1993).

Evidence has been accumulating that CDDP exerts its renal and cochlear toxicity at least in part through oxidative stress, generating reactive oxygen species (ROS; free radicals) and interfering with the antioxidant defense systems of these organs (Appenroth et al, 1993, 1995; Appenroth and Winnefeld, 1993; Clerici et al, 1996; Gabaizadeh et al, 1997; Hannemann and Baumann, 1988; Kameyama and Gemba, 1991; Kopke et al, 1997; Ravi et al, 1995a, 1995b; Sugihara et al, 1987; Sugihara and Gemba, 1986; Zhang and Lindup, 1994). This notion is supported by successful attempts at ameliorating the nephrotoxic or ototoxic side effects in experimental animals in vivo by antioxidant therapy with D-methionine (Campbell et al, 1996, 1999; Li et al, 2001; Reser et al, 1999), diethyldithiocarbamate acid (Rybak et al, 1995), and sodium thiosulfate (Iwamato et al, 1984; Saito et al, 1997). However, such a pharmacologic intervention administered 
systemically has frequently been problematic because of detrimental effects on the efficacy of CDDP against certain tumors (Aamdal et al, 1987; Inoue et al, 1991; Li et al, 2001; Reser et al, 1999). Only local delivery of $\mathrm{D}$-methionine to the round window membrane has been shown to protect hearing from CDDP damage without affecting its chemotherapeutic action on the tumor cells (Li et al, 2001).

In many aspects, the ototoxicity of CDDP resembles that of aminoglycoside antibiotics. The pathology of aminoglycoside-induced hair cell loss shows a similar base-to-apex pattern of sensory cell destruction, and the underlying mechanism includes the formation of reactive oxygen species (Forge and Schacht, 2000; Schacht, 1999; Sha and Schacht, 1999c). Furthermore, aminoglycosideinduced ototoxicity is also limited by the antioxidant state of inner ear tissues. Depletion of glutathione levels through diet aggravates the auditory side effects of both aminoglycoside and CDDP ototoxicity (Lautermann et al, 1995a, 1995b). Consequently, antioxidant therapy can reduce aminoglycoside-induced hair cell loss and threshold shifts, and the same chemicals as in the case of CDDP may be effective, for example D-methionine (Sha and Schacht, 2000). Recently, the interesting suggestion has been made that a drug as simple as salicylate may attenuate aminoglycoside-induced hearing loss (Sha and Schacht, 1999a).

Salicylates are among the most commonly used medicines for their anti-inflammatory, analgesic, antipyretic, and antithrombic properties, mostly in the form of aspirin (acetyl salicylate), which is metabolized to salicylate in serum within 15 to 30 minutes. Although its antiinflammatory actions are primarily based on an inhibition of the enzyme cyclooxygenase and possibly on suppression of cyclooxygenase-2 transcription ( $\mathrm{Xu}$ et $\mathrm{al}$, 1999), salicylate (2-hydroxybenzoate) has several other properties that may contribute to its therapeutic palette. Salicylate can affect the activation of transcription factors, in particular $\mathrm{NF}-\kappa \mathrm{B}$, thereby intervening in apoptotic pathways (Chernov and Stark, 1997; Yin et al, 1998). It is also a hydroxyl radical scavenger, reacting with hydroxyl radicals to form 2,3- and 2,5-dihydroxybenzoic acid in both experimental animals and humans who are experiencing oxidative stress (Ghiselli et al, 1992; O'Connell and Webster, 1990; Powell, 1994). As a case in point, aspirin and salicylate protect against 1-methyl-4phenyl-1, 2, 3, 6-tetrahydropyridine (MPTP)-induced dopamine depletion, a reaction involving free radicals (Aubin et al, 1998). A similar scavenging role may be played by salicylate in the prevention of aminoglycosideinduced hearing loss (Sha and Schacht, 1999a). Furthermore, salicylate and aspirin may have antineoplastic properties (Thun et al, 1993), making these drug even more attractive as a supplemental therapy in cancer treatment.

This study follows the suggestion that salicylate may be potentially useful as a therapeutic intervention to protect against CDDP-induced auditory threshold shifts (Sha and Schacht, 1999b). We determined whether salicylate administration could protect against CDDPinduced ototoxicity, nephrotoxicity, and neurotoxicity, without compromising the antitumor efficacy of CDDP chemotherapy.

\section{Results}

\section{Salicylate Attenuates Acute CDDP-Induced Threshold} Shifts

In the first study, 5 of 15 animals died in the CDDP group and 6 of 14 animals died in the CDDP plus salicylate group, on either the second or third day after the CDDP injection. There were no fatalities among the animals injected with salicylate only. Cisplatin caused a significant hearing loss in the surviving animals, reaching more than $30 \mathrm{~dB}$ at $14 \mathrm{kHz}$ with somewhat lower threshold shifts at $8 \mathrm{kHz}$ (Fig. 1) and $3 \mathrm{kHz}$ (data not shown). The coadministration of salicylate attenuated the threshold shifts significantly to around $10 \mathrm{~dB}$ $(p<0.01)$.
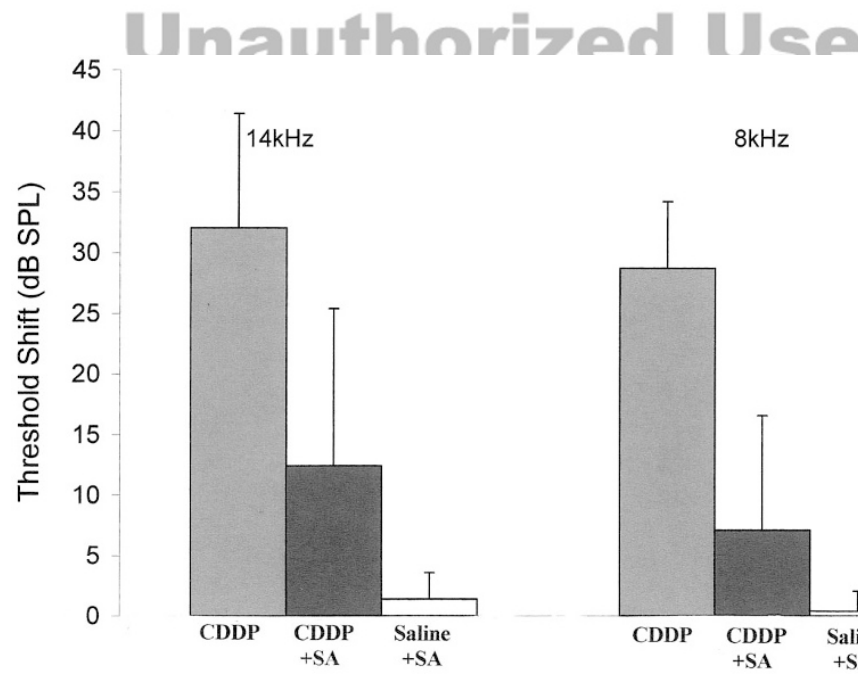


\section{Salicylate Attenuates Auditory Threshold Shifts Caused by Repeated CDDP Dosing}

In this second study, 4 of 10 rats died in the CDDP treatment group (Group II) and 6 of 12 died in the salicylate plus CDDP treatment group (Group IV). Fatalities did not begin to occur in either of these two groups until the day after the third and last injection of CDDP, Day 8. There were no fatalities of either untreated (Group I) or salicylate-treated (Group III) rats.

Administration of a series of three dosages of CDDP was associated with a deterioration of the auditory brain stem response (ABR) at the two highest frequencies tested. In CDDP-treated animals, thresholds at 16 and $24 \mathrm{kHz}$ were significantly elevated at 10 days after CDDP administration compared with threshold values in untreated subjects ( $p<0.05$ ); no ABR changes were evident in response to click stimuli (Fig. 2). These CDDP-induced alterations in ABR thresholds were significantly attenuated by coadministration of salicylate (Fig. 2). Thresholds at $16 \mathrm{kHz}$ and $24 \mathrm{kHz}$ in animals receiving both CDDP and salicylate did not differ from those of untreated animals $(p>0.05)$. Salicylate treatment alone did not produce any significant changes in the ABR thresholds.

\section{Salicylate Protects the Outer Hair Cells from CDDP Damage}

In untreated (Group I) and salicylate-treated (Group III) animals, there was no loss of either inner hair cells or outer hair cells in any of the three cochlear turns examined (Fig. 3, A, C, and E, basal turn; Fig. 4, A and C, middle turn; and Fig. 5, apical turn). In contrast, rats treated with 3 dosages of CDDP (Group II) sustained a mean loss of $20 \% \pm 4 \%$ of their outer hair cells in the basal turn (Fig. 3, B and E), but no significant loss in either their middle ( $p>0.05$, Fig. 4B) or apical turns (Fig. 5). Co-administration of salicylate partially protected the outer hair cells in the basal turn from CDDP-induced damage, reducing the mean loss of outer hair cells to $8 \% \pm 8 \%$ (Fig. 3, D and E; Fig. 5). There was no loss of outer hair cells from either the middle or apical turns of the cochleae in the salicylate plus CDDP-treated rats (Group IV; Fig. 4 and Fig. 5).

The means of the outer hair cell losses in all groups are summarized in Figure 5. Consistent with earlier results (Li et al, 2001; Reser et al, 1999), outer hair cell damage is greatest in the basal turn of the cochlea. The base of the cochlea is also the location where the protection by salicylate is most dramatic.

\section{Salicylate Partially Protects the Kidney against CDDP Damage}

The plasma concentration of blood urea nitrogen (BUN) and creatinine showed large and significant increases after CDDP treatment, even in the additional presence of salicylate (Fig. 6). However, salicylate afforded some protection because the plasma levels of BUN and creatinine in Group IV (salicylate + CDDP)

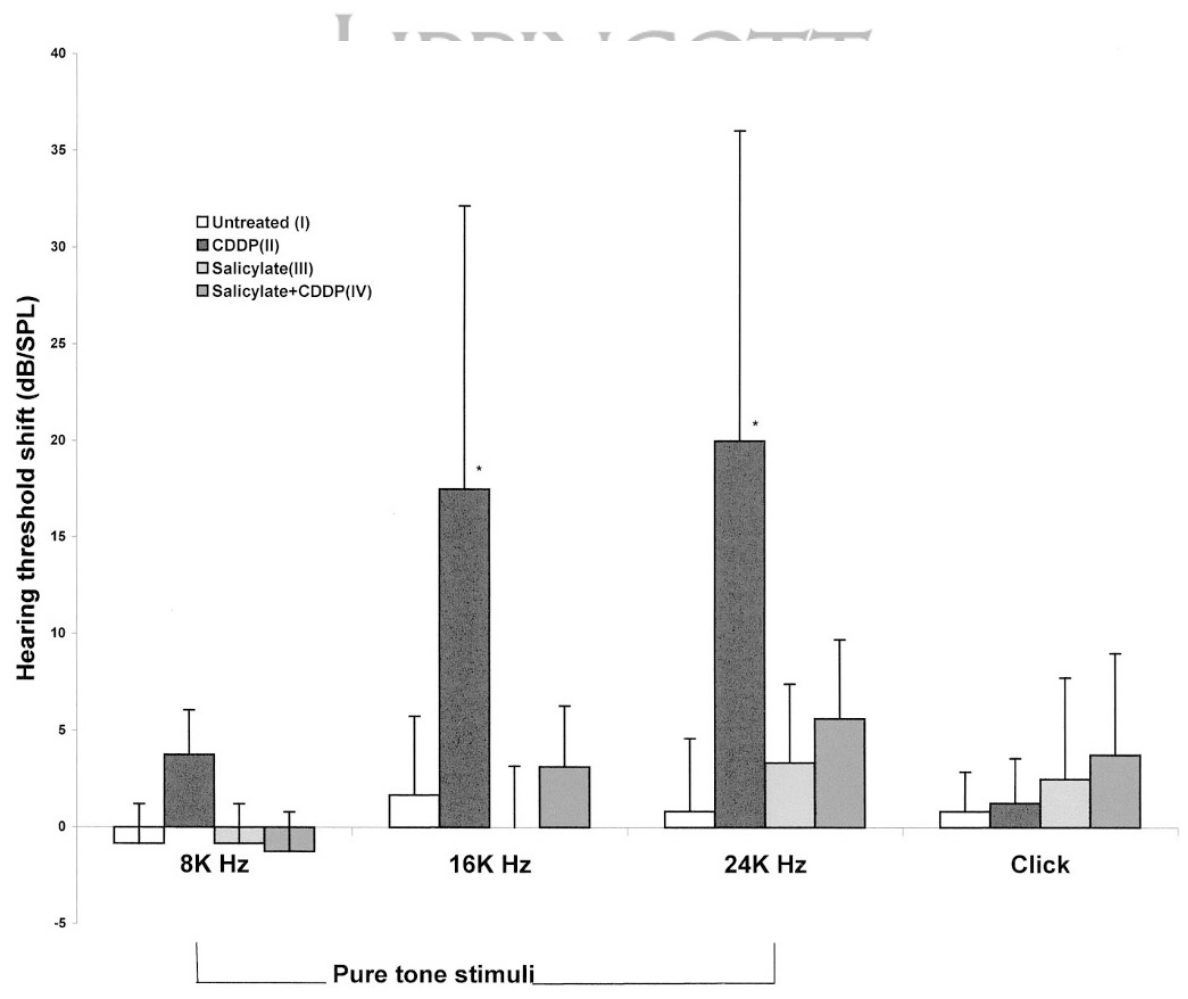

Figure 2.

Salicylate treatment protects against multidose CDDP-induced threshold shifts. Auditory threshold shifts in response to click and 8-24 kHz pure tone stimuli were calculated as the difference between ABR thresholds on the first day of salicylate injection and 10 days after the initial CDDP dose. Measurements were taken at equivalent time periods in the untreated rats. There were significant ABR threshold changes in response to 16 and $24 \mathrm{kHz}$ pure tone stimuli in the CDDP-treated (Group II) rats $(p<0.05)$. There were no significant ABR threshold changes in the animals of the other groups-Group I (untreated), Group III (salicylate only), and Group IV (salicylate + CDDP) $(p>0.05)$. 

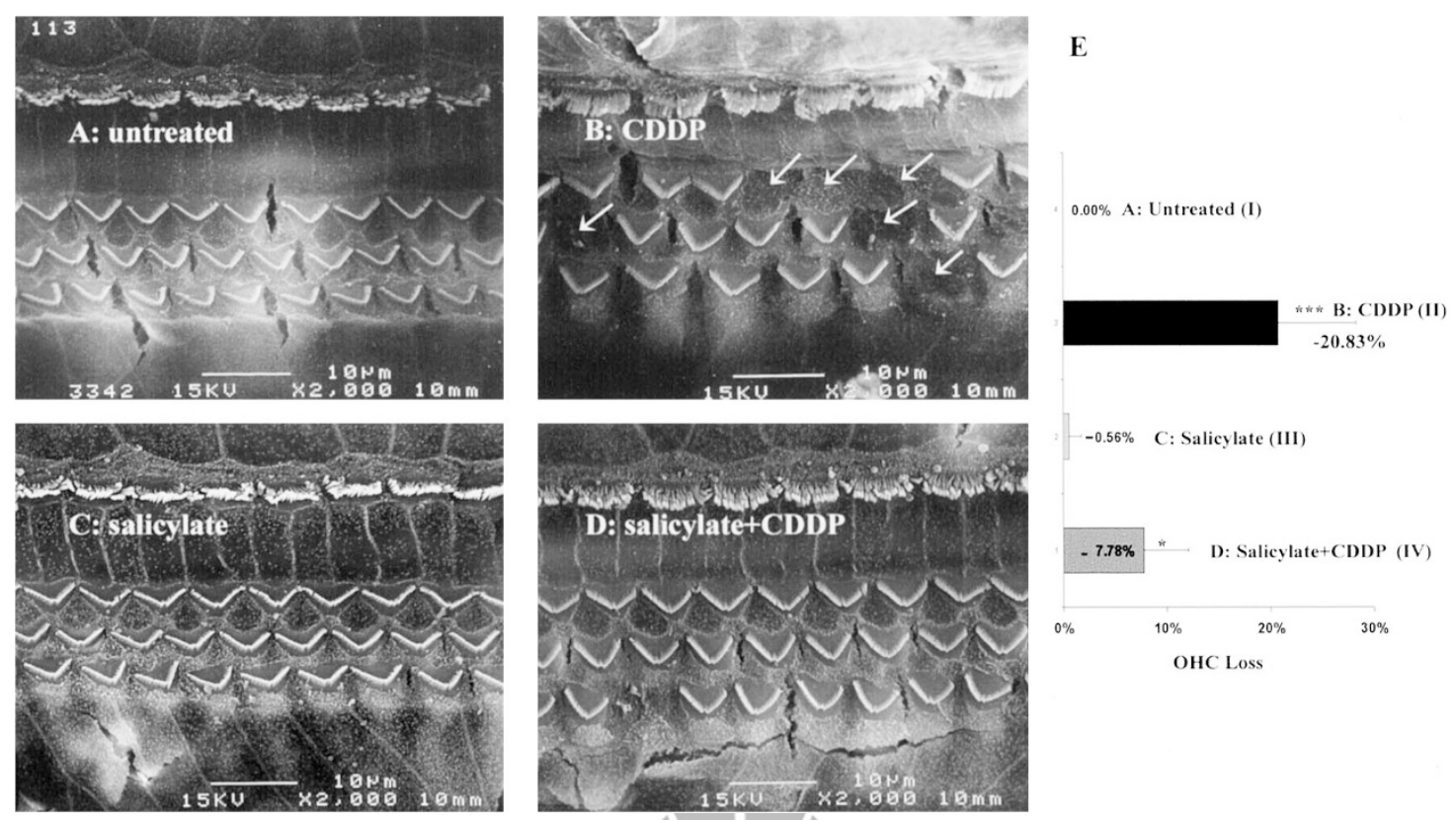

Figure 3.

Salicylate treatment protects outer hair cells in the basal turn from CDDP-induced damage. Scanning electron micrographs (A-D) and a histogram of percentage of outer hair cell loss (E) are presented for: A, controls (Group I); B, CDDP-treatment (Group II); C, salicylate only (Group III); D, salicylate + CDDP (Group IV). Untreated (Group I) and salicylate only (Group III) animals showed a normal configuration of three rows of outer hair cells. In contrast, the CDDP-treated animals had a highly significant loss of outer hair cells (arrows) from the basal turn of the cochlea $(p<0.001)$. Animals treated with salicylate before and during CDDP therapy showed only a slight loss of outer hair cells from the basal turn $(p<0.05)$. E, a comparison of outer hair cell counts from CDDP-treated animals (Group II) and animals treated with CDDP + salicylate (Group IV) showed a significant protective effect of salicylate against CDDP-damage $(p<0.05) .{ }^{* *} p<0.001,{ }^{\star} p<0.05$. Calibration bars, $10 \mu \mathrm{m}$.

were significantly lower $(p<0.05)$ than in the Group II (CDDP alone) rats.

\section{Salicylate Affords Minimal Protection of the Motor Nerve Amplitude from CDDP-Induced Changes}

In the third study of animals there were no fatalities in any of the four groups of rats (Groups la to IVa); nerve conduction parameters were tested 3 days after the second injection of cisplatin. Administration of CDDP alone (Group Ila) induced a significant slowing of both sensory and motor conduction velocity $(p<0.05$ and 0.01 , respectively). The velocity in the caudal and tibial nerves was also significantly diminished in Group IVa receiving salicylate plus CDDP (Fig. $7 ; p<0.01$ and $p<0.01$, respectively). There were no changes in conduction velocity in animals receiving either saline or salicylate alone.

The magnitude of change in conduction velocity was remarkably consistent for the groups receiving CDDP with or without additional salicylate $(16 \%$ versus $15 \%$ for caudal nerve; $38 \%$ versus $39 \%$ for the tibial nerve). In contrast, the reduction in motor amplitude by CDDP was partially prevented by additional dosing with salicylate (Fig. 8). The amplitude of the compound muscle action potential (CMAP) was reduced by approximately $30 \%$ in Group Ila ( $p=0.03$ compared with controls) but by less than 10\% in Group IVa. This difference between groups IIa and IVa was significant ( $p=0.03$ ). No significant effects were observed for the sensory nerve amplitude.

\section{Salicylate Does Not Interfere with the Chemotherapeutic} Action of Cisplatin

The MTLn3 breast cancer tumors in both the Group I (untreated) animals and Group III (salicylate-treated) animals showed a pattern of unlimited growth, increasing from a normalized value of 1 to 2.8 (0.8 in Group I and to $3.0 \pm 1.0$ in Group III). In contrast, the tumors in both the Group II (CDDP) and Group IV (salicylate + CDDP) rats were well-controlled and decreased from 1 to $0.008 \pm 0.006$ in Group II and from 1 to $0.015 \pm 0.02$ in Group IV ( $p<0.001$, Fig. 9). In most cases, the breast cancer tumors in both the Group II and Group IV animals were no longer detectable after the sequence of three CDDP treatments, 15 $\mathrm{mg} / \mathrm{kg}$ aggregate dose.

Both palpable metastasis and histologic evidence of tumor cell metastasis were present in $33 \%$ of the untreated (Group I) animals and in $40 \%$ of the salicylate-treated (Group III) animals. In contrast, no palpable or histologic metastasis of MTLn3 breast cancer cells was observed in either the Group II (CDDP) or Group IV (salicylate + CDDP)-treated animals.

\section{Discussion}

The attenuation of CDDP-induced ototoxic and nephrotoxic side effects by salicylate is a new and potentially powerful application of this remarkable drug. CDDP is an indispensable antitumor agent, and currently no clinical treatment exists to prevent its toxicity 

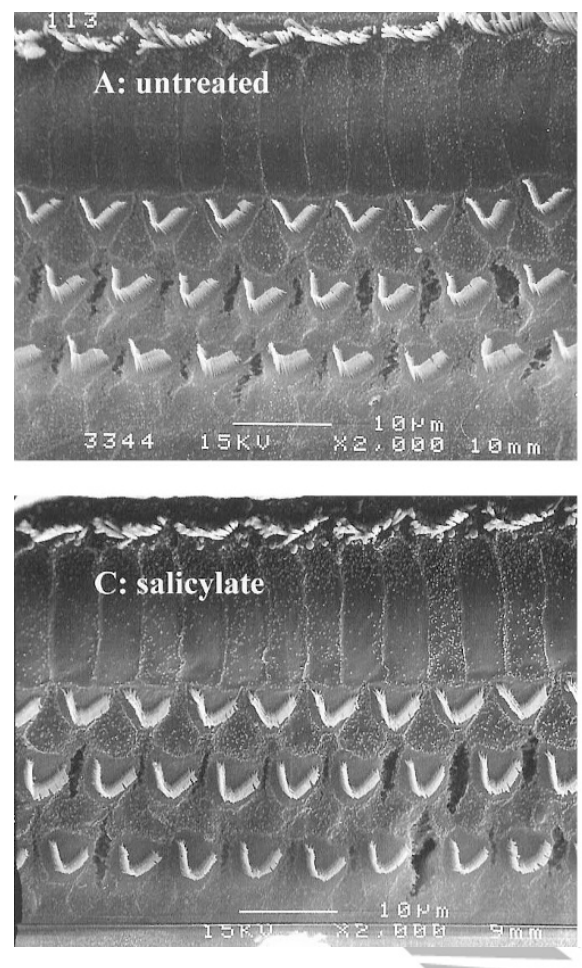

Figure 4.

Cisplatin treatment does not affect the integrity of outer hair cells in the middle turn of the cochlea. Scanning electron micrographs show intact outer hair cells in the middle turn of the cochleae in all treatment groups and controls. A, Untreated controls (Group I); B, CDDP-treatment (Group II); C, Salicylate only (Group III); D, Salicylate + CDDP (Group IV). Calibration bars, $10 \mu \mathrm{m}$.

to the inner ear. In animals, systemic administration of either L- or D-methionine before CDDP treatment does indeed reduce both the ototoxic and nephrotoxic side effects. However, there is also an unwanted partial tumor-sparing effect of systemic methionine treatment on the oncolytic action of cisplatin ( Li et al, 2001; Reser et al, 1999). Similar detrimental effects on the chemotherapeutic efficacy of CDDP treatment have been reported for sodium thiosulfate cotreatment (Aamdal et al, 1987; Inoue et al, 1991). It is likely that both methionine and sodium thiosulfate have a similar primary mode of "protective" action through the direct binding of CDDP by their thiol groups. This binding and resulting inactivation of CDDP probably occurs before entry into cells. Salicylate, in contrast, does not affect the antitumor efficacy of CDDP.

Both the morphologic assessment of hair cell loss and the physiologic measure of hearing acuity (using ABR) showed attenuation by salicylate of CDDP ototoxicity, indicating parallel protection at both structural and functional levels. CDDP induces complex morphologic changes to the cochlea, which consistently include extensive damage to outer hair cells, especially in the basal turn of the cochlea (Kamimura et al, 1999; Stadnick et al, 1975). Loss of basal outer hair cells was clearly attenuated by salicylate, whereas cells in the apical and middle turns remained largely unaffected by CDDP. We also observed some animalto-animal variability with respect to the extent of damage, similar to clinical studies in which not all patients receiving CDDP chemotherapy experienced a profound hearing loss (Laurell and Jungnelius, 1990).
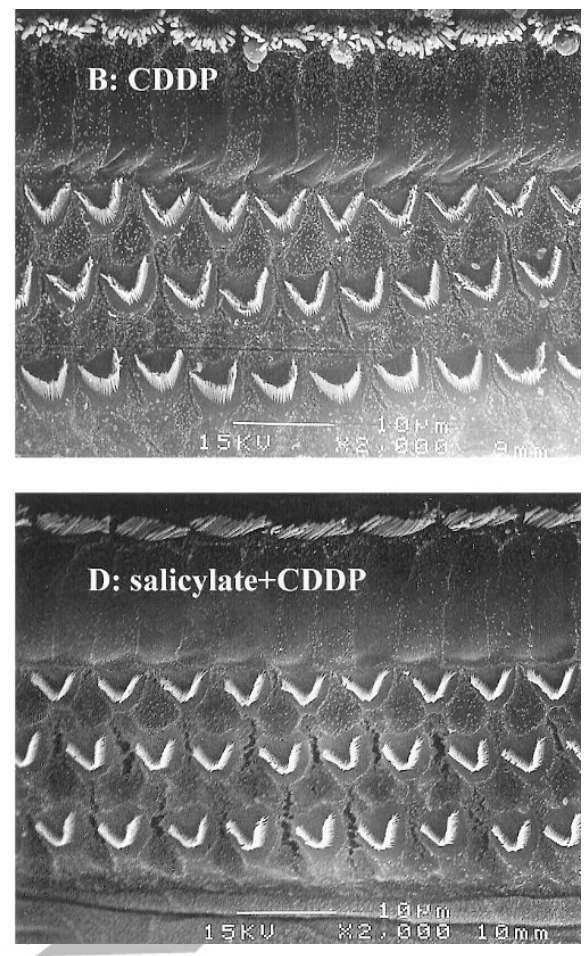

The

Nephropathy is another main side effect of CDDP chemotherapy (Doyban et al, 1980; Gonzalez-Vitale et al, 1977). The present clinical strategy to prevent CDDP-induced nephropathy is hydration; some antioxidants also show protective effects on the kidney against CDDP (Appenroth et al, 1993; Appenroth and Winnefeld, 1993; Basinger et al, 1990; Jones and Basinger, 1989; Matsushima et al, 1998). In this study CDDP treatment induced a decline in kidney function in CDDP-treated animals, as evidenced by significant increases in the plasma levels of BUN and creatinine. Salicylate treatment demonstrated a partial protection of kidney function against damage initiated by CDDP chemotherapy, suggesting that at least some of the nephrotoxic actions of CDDP are similar to the ototoxic actions.

Although the precise mechanism of cytotoxicity of CDDP is not known, we can speculate that the action of salicylate may be based on two factors. First, salicylate is a radical scavenger (Dinis et al, 1994), and there is compelling evidence that the formation of free radicals by CDDP contributes to its toxicity (Appenroth et al, 1993, 1995; Clerici et al, 1996; Freeman and Crapo, 1982; Hannemann and Baumann, 1988; Kameyama and Gemba, 1991; Kopke et al, 1997; Ravi et al, 1995; Rybak et al, 1999; Sugihara and Gemba, 1986; Sugihara et al, 1987; Zhang and Lindup, 1994). Such a mechanism would be akin to the protection by salicylate against MPTP-induced dopamine depletion in mice (Aubin et al, 1998), and the prevention of aminoglycoside-induced ototoxicity (Sha and Schacht, 1999a). 


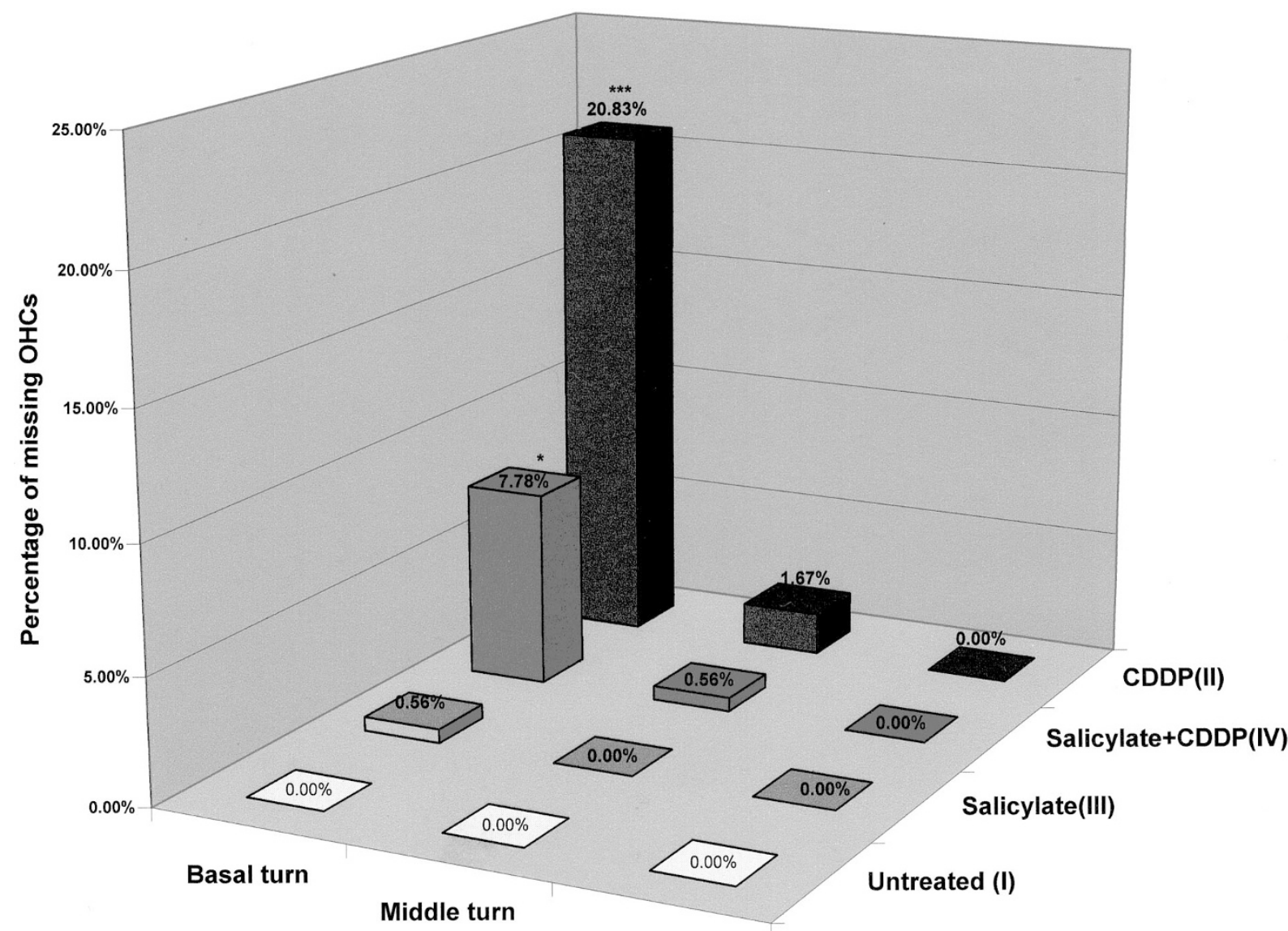

Apical turn

Figure 5.

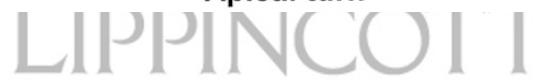

Cytocochleograms of outer hair cell integrity demonstrate the otoprotective effect of salicylate treatment. This quantitative representation of outer hair cell loss shows the loss of outer hair cells from the basal turn of the cochlea after cisplatin treatment and its partial prevention by cotreatment with salicylate. There was no significant loss of outer hair cells from the apical and middle turns in the cochleae of any of the experimental groups. ${ }^{* * *} p<0.001$; ${ }^{*} p<0.05$.

Secondly, cisplatin-exposed cells have been shown to increase the ability of NF- $\kappa$ B to bind DNA (Maldonado et al, 1997), and genes regulated by NF- $\kappa$ B are involved in the control of apoptotic processes (Pomerantz and Baltimore, 2000; Vollgraf et al, 1999). In the cochlea, exposure to ototoxic levels of CDDP promotes rapid translocation of $\mathrm{NF}-\kappa \mathrm{B}$ to the nucleus and a subsequent increase in the expression level of the A1 adenosine receptor, which plays a role in the antioxidant defenses of inner ear sensory cells (Nie et al, 1998). Both aspirin and salicylate have the capacity to inhibit the translocation of NF- $\kappa \mathrm{B}$ to the nucleus by their action on $\mathrm{I}_{\kappa} \mathrm{B}$ kinase-(Yin et al, 1998) and may thus interact with a CDDP-action on apoptotic pathways. Furthermore, cancer cells are more susceptible to chemotherapy when the activation of $\mathrm{NF}-\kappa \mathrm{B}$ is inhibited (Wang et al, 1996); therefore, salicylate treatment may also aid the oncolytic action of CDDP.

In contrast to the beneficial effects of salicylate on the inner ear and the kidney, there was only minimal protection of CDDP-induced changes in peripheral nerve function. The induced reduction in the CMAP was somewhat lessened, but the CDDP-induced slowing of maximal conduction velocity was unaltered by salicylate. The maximal conduction velocity reflects activity of the largest myelinated neurons, whereas the peak of the CMAP also includes activity in smaller diameter and slower conducting axons (Arezzo, 1997). The acute alteration in velocity observed in this study may reflect a toxic neuronopathy as well as a toxic axonopathy (Schaumburg et al, 1992). The antiinflammatory and hydroxyl radical scavenger properties of salicylate may partially prevent the neurotoxic side effects limited to the distal axons, but may be unable to alter pathologic changes that involve the somas of peripheral neurons. It is also possible that the mechanism of damage in the peripheral nervous system differs from CDDP's damaging effect on the inner ear and the kidney, a notion difficult to evaluate in the absence of concrete information on the mechanisms of CDDP toxicity.

One of the problems with current animal models of CDDP ototoxicity is the high systemic toxicity of the drug. Mortality is high in the experimental protocols that are required to produce a significant hearing loss in either rats or mice. Within several days of receiving either a single high dose or the last dosage of the cumulative series of three CDDP injections, the mortality of the treated rats ranged from $33 \%$ to $50 \%$. Similar rates have been observed in other studies 


\section{Figure 6.}

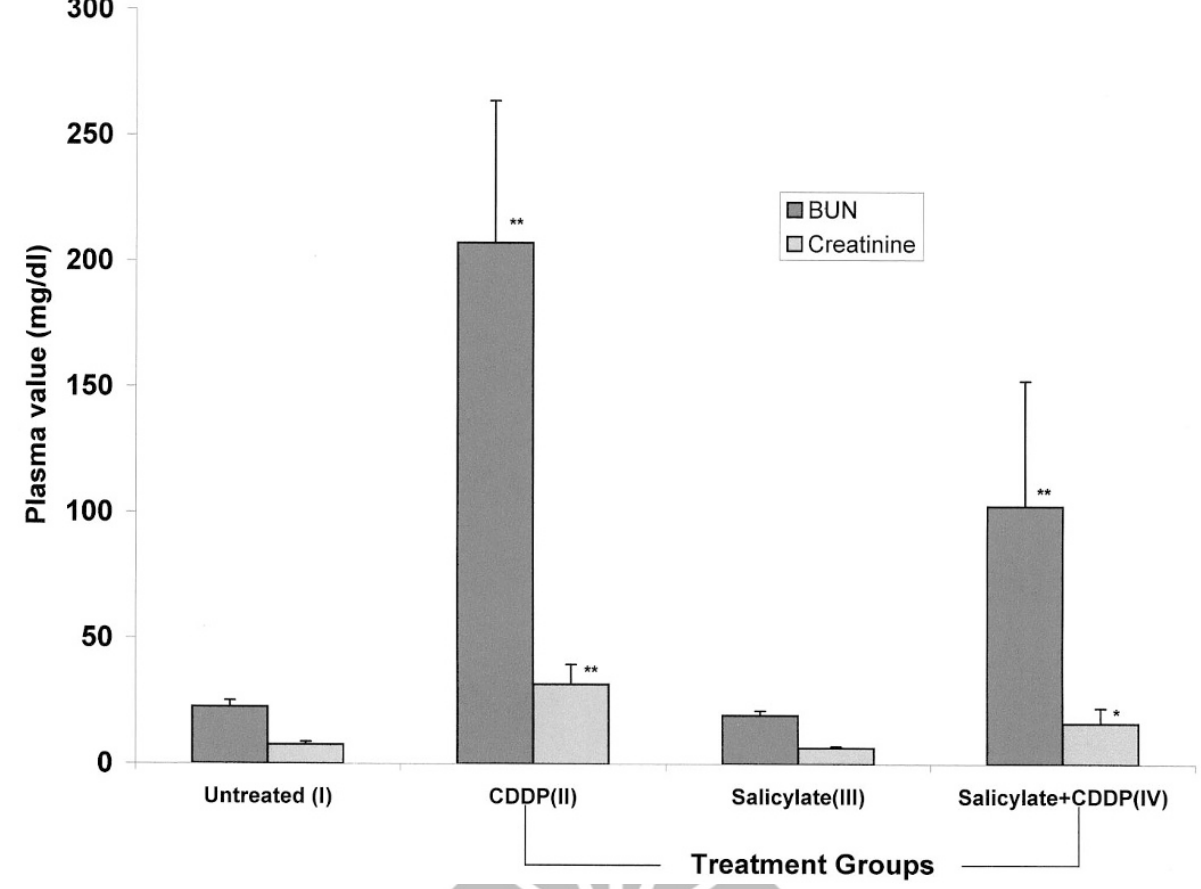

Salicylate treatment partially protects renal function from CDDP-induced nephrotoxicity. Blood urea nitrogen (BUN) and creatinine plasma concentrations were measured at the end of CDDP treatment. The BUN and creatinine concentrations were significantly elevated in animals treated with CDDP alone (Group II) and with salicylate $+\operatorname{CDDP}$ (Group IV) compared with controls. There was also a significant difference between these two treatment groups $(p<0.05)$, showing that salicylate treatment provided a significant partial protection of kidney function against CDDP-induced damage.

(Campbell et al, 1996; Li et al, 2001; Ravi et al, 1995a, 1995b; Reser et al, 1999; Rybak, personal communication). The systemic toxicity of CDDP, which may result from malnutrition, electrolyte imbalance, and metabolic acidosis (Schweitzer, 1993), remained ūnchanged in the salicylate plus CDDP-treated rats. This again may point to differences in the underlying mechanisms. Importantly, however, the protection provided by salicylate treatment was effective under both experimental conditions, that is, a high-dose acute administration of CDDP in nontumor bearing (normal) rats and a regimen of divided dosing of CDDP in rats bearing breast cancer tumors.

Finally, potential side effects of an aspirin or salicylate treatment on auditory function should be considered. Both drugs have ototoxic potential at high doses resulting in temporarily elevated thresholds and tinnitus. On cessation of aspirin/salicylate dosing, however, hearing returns to normal, and there is no confirmed report of any permanent auditory damage by salicylate (Garetz and Schacht, 1996). Consistent with the complete reversibility of salicylate ototoxicity, no hearing loss was measured in animals that received salicylate alone. Gastrointestinal bleeding is also a concern in the chronic administration of high doses of aspirin or salicylate and was indeed observed in approximately $80 \%$ of the animals in the salicylatetreated groups. The additional administration of a proton-pump blocker should be considered to counteract this side effect.

In summary, these results suggest that salicylate cotreatment may have clinical application for prevent- ing the ototoxicity and attenuating the kidney damage induced by CDDP therapy without compromising its effectiveness as an antineoplastic agent.

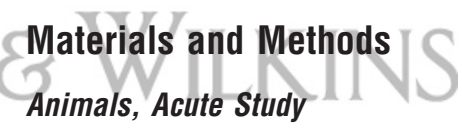

A preliminary study was conducted using an acute (single-injection) model of CDDP toxicity (Ravi et al, 1995a). Male Wistar rats (Charles River Laboratories, Wilmington Massachusetts; body weight 350 to $500 \mathrm{~g}$ ) had free access to water and food and were divided into three groups (Group I: controls receiving saline and salicylate, $n=5$; Group II: CDDP, $n=10$; Group III: CDDP + salicylate, $n=8$ ). Cisplatin was dissolved in saline to a concentration of $1 \mathrm{mg} / \mathrm{ml}$ and infused intraperitoneally at a dose of $16 \mathrm{mg} / \mathrm{kg}$ body weight over 30 minutes. Sodium salicylate was given subcutaneously twice daily each time at a dosage of 100 $\mathrm{mg} / \mathrm{kg}$ body weight, starting 1 day before the injection of CDDP and continuing for 4 days thereafter. The protocols on animal use were approved by the University of Michigan's Committee on Use and Care of Animals. Animal care for this part of the experiments was under the supervision of the University of Michigan's Unit for Laboratory Animal Medicine.

\section{Auditory Threshold, Acute Study}

Shifts in hearing thresholds were assessed by ABR. Each rat had its threshold determined before the study and then 4 days after CDDP treatment. Animals were 
A

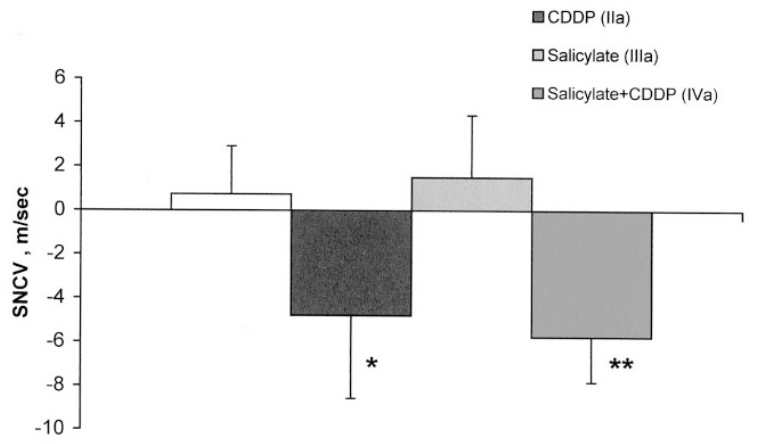

B

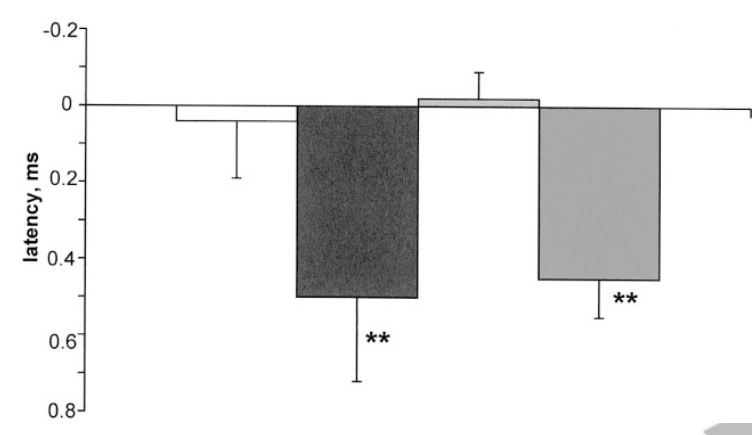

Figure 7.

Salicylate does not protect against the CDDP-induced decrease in conduction velocity in caudal and tibial nerves. A, Caudal nerve conduction velocity was measured at the baseline and 7 days after CDDP treatment had started $(n=$ 5). B, CMAP onset latencies in tibial nerve measured at the baseline and 7 days after CDDP treatment had started $(n=5)$. The decrement in sensory and motor conduction velocity was statistically significant for CDDP-treated (Group II) and salicylate + CDDP-treated animals (Group IV) compared with untreated animals (Group I). Vertical lines indicate standard deviations. * $p<0.05$; ** $p<0.01$.

anesthetized with an intraperitoneal injection of ketamine $(70 \mathrm{mg} / \mathrm{kg}$ body weight) and xylazine $(7 \mathrm{mg} / \mathrm{kg}$ body weight). Tone bursts of 8 and $14 \mathrm{kHz}(10 \mathrm{~ms}$ duration, $1 \mathrm{~ms}$ rise/fall time) were generated using a Fordham Audio Generator (Model AG-298, Fordham Radio Supply, Hauppauge, New York) and delivered to the left external auditory meatus in a closed acoustic system through an ear bar connected to a Beyer DT-48 transducer (Beyer Dynamic, Farmingdale, New York). The output was fed to an amplifier, viewed on an oscilloscope, and recorded. The average responses from 1,024 stimuli were obtained for each frequency by reducing the sound intensity in 10-dB steps and finally at 5-dB intervals near threshold. Threshold was defined as the lowest stimulus level at which a positive waveform in the evoked response was evident. Thresholds at each frequency were verified at least twice.

\section{Animals, Chronic Studies}

Based on preliminary results, a second study was designed with a total of 40 female 7 -week-old tumorbearing Fisher344 rats. Twenty rats carrying a MTLn3 breast cancer tumor of $1-$ to $2-\mathrm{cm}$ diameter were

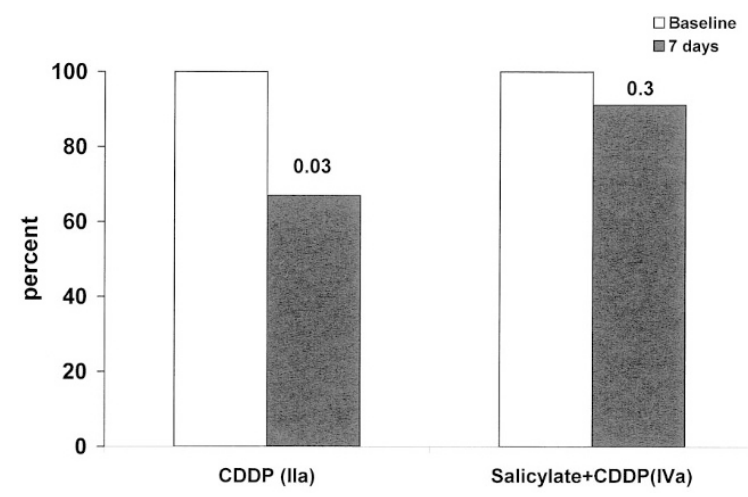

Figure 8.

Salicylate partially protects from the CDDP-induced decrease in compound muscle action potential (CMAP) amplitude in the tibial nerve. CMAP amplitude in tibial nerve measured on seventh day after the start of CDDP treatment. The difference from control values was statistically significant $(p=0.03)$ in CDDP-treated animals (Group II), but not in animals receiving salicylate + CDDP (Group IV; $p=0.3$ ). Data are shown normalized to the baseline value. Numbers above the bars indicate $p$ values.

divided into four groups: Group I, untreated $(n=3)$; Group II, CDDP ( $n=6 ; 5 \mathrm{mg}$ of CDDP $/ \mathrm{kg}$ ip each at Days 1, 4, and 7); Group III, salicylate $(n=5 ; 100$ $\mathrm{mg} / \mathrm{kg}$, sc, bid); Group IV, salicylate + CDDP $(n=6)$. In a third study, another 20 rats without tumor implantation were divided into the four treatment groups (Groups la to IVa) described above for the assessment of neurotoxicity. All animals were housed in an American Association for the Accreditation of Laboratory Animal Care (AAALAC)-accredited animal facility, and all procedures were in accordance with the laboratory animal care and use guidelines of the National Institutes of Health and those of the Albert Einstein College of Medicine Internal Animal Care and Use Committee.

\section{Tumor Cell Injection and Measurement of Tumor Growth}

Rats were inoculated with MTLn3 malignant breast tumor cells, a clinically relevant animal model for metastatic mammary adenocarcinoma (Farina et al, 1998; Li et al, 2001; Reser et al, 1999). A single injection of $0.15 \mathrm{ml}$ of a tissue culture medium containing $1 \times 10^{6}$ MTLn3 cells was given subcutaneously into the fat pad between the third and fourth nipples. The drug treatments were initiated only after the implanted breast cancer cells reached a tumor size between 1 and $2 \mathrm{~cm}$ in diameter. Tumors growing at the site of injection were measured before each CDDP injection in animals of Groups II and IV and at corresponding times in Group I and III animals, and again before they were killed. Increases or decreases in tumor size were determined by comparing a tumor's size on Day 10 with its size on Day 0. At the time of tumor measurement, the abdominal areas and local lymph nodes of the rats were palpated to detect tumor metastasis. At the end of the study, a complete autopsy that included the sectioning of lungs, liver, kidneys and small intestines was performed on all animals to detect nonpalpable metastasis of the MTLn3 tumor cells. 


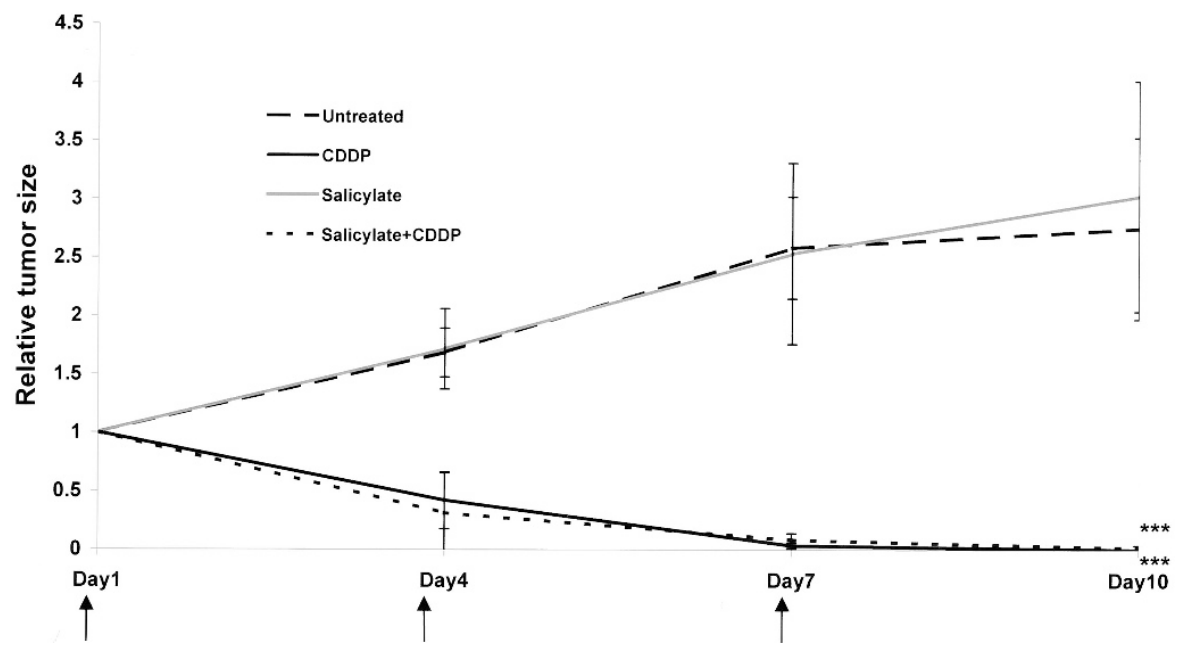

Figure 9.

Cotreatment with salicylate does not affect CDDP-control of MTLn3-initiated tumors. Differences in tumor volume of MTLn3-initiated breast cancers tumors in response to CDDP treatment alone, salicylate treatment alone, or CDDP-treatment with salicylate were compared with the unrestricted tumor growth in untreated animals. Days of CDDP injection are indicated by arrows. The tumor size in untreated (Group I) and salicylate-only treated animals (Group III) was significantly greater $(p<0.001)$ than in either of the CDDP-treated groups (Group II, CDDP only; Group IV, salicylate + CDDP). ${ }^{* * *} p<0.001$.

\section{ABR Recordings, Second Study}

ABRs were recorded before the first injection of salicylate and 10 days after treatment in Group III and IV animals, and at the equivalent times in Group I and II animals. ABRs were recorded under general anesthesia (ketamine $35 \mathrm{mg} / \mathrm{kg}$ and xylazine $15 \mathrm{mg} / \mathrm{kg}$; im), using a Tucker-Davis Technology system (TDT System II) and a Gateway 2000 Pentium II computer (model E3110). Stimuli were digitally synthesized using Siggen software and presented through an insert earphone (Etymotic Research, ER-2). Click and tōne pip acoustic stimuli from 8 to $24 \mathrm{kHz}$ were presented at octave intervals. The intensity of the acoustic stimuli pre-ABS responses was expressed as a decibel (dB) sound/pressure level peak equivalent. Animals were presented with a stimulus intensity series, progressively lowered in 10-dB decrements. Each averaged signal consisted of 500 stimulus presentations, with a $10 \mathrm{~ms}$ analysis time. Electrical activity was recorded via a platinum needle electrode (Grass Instruments) inserted into the scalp at the vertex, referenced to another needle electrode in a deep neck muscle. A third needle electrode in the pinna served as a ground. Intensities that seemed to be at or near threshold were repeated. Threshold was defined as the lowest intensity capable of producing a visually detectable, reproducible $A B R$ response and values were extrapolated to the nearest $5 \mathrm{~dB}$ assuming a log-linear fall-off with intensity. At threshold, the baseline-to-peak amplitude of the largest $A B R$ component was reduced to less than $10 \%$ of that evoked by a $90-\mathrm{dB}$ stimulus.

\section{Electrophysiologic Recording of Sensory and Motor Nerves}

Electrophysiologic data were recorded from a total of 20 female Fischer 344 rats approximately the same age and weight as those used for assessment of ototoxicity and nephrotoxicity. Animals used for electrophysiology were tumor-free throughout the study period. Animals were randomly assigned to one of four groups ( $n=5$ per group) with treatment identical with those used in the other studies. Data were collected under general anesthesia (combination of ketamine 35 $\mathrm{mg} / \mathrm{kg}$ and xylazine $15 \mathrm{mg} / \mathrm{kg}$, im), using a TecaTD-10 instrument and previously outlined methods (Apfel et al, 1993). Briefly, sensory nerve conduction velocity was measured orthodromically on the distal caudal nerve. Subcutaneous platinum needle electrodes (Grass Instruments) were used for both stimulation and recording. Supramaximal stimulation was achieved using a constant voltage, $0.1 \mathrm{~ms}$ square pulse. The active recording lead was positioned 60 $\mathrm{mm}$ proximal to the stimulating cathode and $5 \mathrm{~mm}$ distal from the reference electrode. Both pairs of electrodes were located on the midline of the dorsal tail. Sensory response amplitude was determined from baseline to peak of the initial depolarization and, generally, three to five responses were averaged. Maximal motor conduction velocity was estimated from the onset latency of the CMAP in the intrinsic muscles of the distal plantar surface of the foot after supramaximal stimulation of the tibial nerve at the ankle. Motor amplitude was determined from baseline to the peak of the CMAP. During all recording sessions, animals were positioned on a circulating warm water pad and rectal temperature was monitored.

In each animal, electrophysiologic data were assessed at baseline, immediately before the initial dose of CDDP, and 7 days after the onset of CDDP administration (immediately before the final dose of cisplatin). Thus, at the final electrophysiologic recording session, animals in Groups lla had received a cumulative dose of $10 \mathrm{mg} / \mathrm{kg}$ of CDDP, animals in Group IIla had received 9 days of salicylate, and animals in 
Group IV had received a cumulative dose of $10 \mathrm{mg} / \mathrm{kg}$ of CDDP and 9 days of salicylate.

\section{Renal Function}

Function was assessed by measurements of BUN and creatinine levels in the plasma. A 0.3- to $0.4-\mathrm{ml}$ aliquot of blood was collected from the caudal vein under general anesthesia. Control samples of blood were taken before the first administration of salicylate and again 10 days after the onset of CDDP administration or at the corresponding times for untreated animals. The concentrations of BUN and creatinine in the blood plasma were measured at the Clinical Medicine Laboratory of the Albert Einstein College of Medicine.

\section{Scanning Electron Microscopy}

After the final ABR recordings and blood samples were obtained, the anesthetized animals were perfused intracardially with $4 \%$ paraformaldehyde in 0.1 cacodylate buffer ( $\mathrm{pH}$ 7.4). The temporal bones were removed, and the perilymphatic space of each cochlea was gently perfused with $2.5 \%$ glutaraldehyde in $0.1 \mathrm{M}$ cacodylate buffer $(\mathrm{pH} 7.4)$ via cochleostomies at the round and oval windows. Bony capsules were removed to expose the organ of Corti, after which the specimens were post-fixed overnight at $40^{\circ} \mathrm{C}$ in the $2.5 \%$ glutaraldehyde fixative solution. Specimens were then washed 3 times in phosphate buffer and post-fixed in $1 \%$ osmium tetroxide for 1 hour at $40^{\circ} \mathrm{C}$. The osmicated organ of Corti specimens were dehydrated through a graded series of ethanol and criticalpoint-dried using liquid carbon dioxide at the Analytical Imaging Facility of the Albert Einstein College of Medicine. Critical-point-dried organ of Corti specimens were attached to aluminum scanning electron micrograph stubs with aluminum paint and then sputter coated with gold-palladium. The surface of all organ of Corti specimens was examined in a JSM6400 JEOL scanning electron microscope operating at 15 kV. Representative segments of each organ of Corti specimen were analyzed at the basal, middle, and apical turns. The scanning electron micrographs used for cell counts were taken from areas that were approximately the same anatomical distance from the round window of each animal. Outer hair cell and inner hair cell numbers were determined by counting the number of intact hair cell cuticular plates. The length of the cochlear duct containing 10 inner hair cells was assumed to correlate with a population of 30 outer hair cells. This method of normalization was chosen because only outer hair cells were lost during CDDP treatment, whereas inner hair cell cuticular plates with stereociliary bundles were always present in all cochlear turns of even the most severely CDDPdamaged specimens.

\section{Statistical Methods}

All comparisons of data for statistical significance were performed by ANOVA with the resultant $F$ values converted to $p$ values and reported as significant at either the $5 \%$ level $(p<0.05)$, the $1 \%$ level $(p<0.01)$, or as highly significant at the $0.1 \%$ level $(p<0.001)$.

\section{References}

Aamdal S, Fodstad O, and Pihl A (1987). Some procedures to reduce cis-platinum toxicity reduce antitumor activity. Cancer Treat Rev 14:389-395.

Apfel SC, Arezzo JC, Moran M, and Kessler JA (1993). Effects of administration of ciliary neurotrophic factor on normal motor and sensory peripheral nerves in vivo. Brain Res 604:1-6.

Appenroth D and Winnefeld K (1993). Role of glutathionine for cisplatin nephrotoxicity in young and adult rats. Ren Fail 15:135-139.

Appenroth D, Winnefeld K, Schroter H, and Rost M (1993). Beneficial effects of acetylcysteine on cisplatin nephrotoxicity in rats. J Appl Toxicol 13:189-192.

Appenroth D, Gambaryan S, Winnefeld K, Leiterer M, Fleck C, and Brăunlich H (1995). Functional and morphological aspects of thallium-induced nephrotoxicity in rats. Toxicology 96:203-215.

Arezzo JC (1997). The use of electrophysiology for the assessment of diabetic neuropathy. Neurosci Res Com 21: 13-22.

Aubin N, Curet O, Deffois A, and Carter C (1998). Aspirin and salicylate protect MPTP-induced dopamine depletion in mice. J Neurochem 71:1635-1642.

Basinger MA, Jones MM, and Holscher MA (1990). L-methionine antagonism of cis-platinum nephrotoxicity. Toxicol Appl Pharmacol 103:1-15.

Blakely BW, Gupta AK, Myers SF, and Schwan S (1994). Risk factors for ototoxicity due to cisplatin. Arch Otolaryngol Head Neck Surg 120:541-546.

Campbell KC, Meech RP, Rybak LP, and Hughes LF (1999). D-Methionine protects against cisplatin damage to the stria vascularis. Hear Res 138:13-28.

Campbell KCM, Rybak LP, Meech RP, and Hughes L (1996). D-methionine provides excellent protection from cisplatin ototoxicity in the rat. Hear Res 102:90-98.

Chernov MV and Stark GR (1997). The p53 activation and apoptosis induced by DNA damage are reversibly inhibited by salicylate. Oncogene 14:2503-2510.

Clerici WJ, Hensley K, DiMartino DL, and Butterfield DA (1996). Direct detection of ototoxicant-induced reactive oxygen species generation in cochlear explants. Hear Res 98:116-124.

Dinis TC, Maderia VM, and Almeida L (1994). Action of phenolic derivatives (acetaminophen, salicylate, and 5-aminosalicylate) as inhibitors of membrane lipid peroxidation and as peroxyl radical scavengers. Arch Biochem Biophys 315:161-169.

Doyban DC, Levi J, Jacobs C, Kosek J, and Weiner MW (1980). Mechanisms of cisplatin-induced nephrotoxicity: II. Morphologic observations. J Pharmacol Exp Ther 213:551556.

Farina KL, Wyckoff JB, Rivera J, Lee H., Segall JE, Condeelis JS, and Jones JG (1998). Cell motility of tumor cells visualized in living intact primary tumors using green fluorescent protein. Cancer Res 58:2528-2532. 
Forge A and Schacht $\mathrm{J}$ (2000). Aminoglycoside antibiotics. Audiol Neurootol 5:3-22.

Fram RJ (1992). Cisplatin and platinum analogues: Recent advances. Curr Opin Oncol 4:1073-1079.

Freeman BA and Crapo JD (1982). Biology of disease: Free radicals and tissue injury. Lab Invest 47:412-426.

Gabaizadeh R, Staecker H, Liu W, and Van De Water TR (1997). BDNF protection of auditory neurons from cisplatin involves changes in intracellular levels of both reactive oxygen species and glutathione. Mol Brain Res 71-78.

Garetz SL and Schacht J (1996). Ototoxicity: Of mice and men. In: Van De Water TR, Popper AN, and Fay RR, editors. Clinical aspects of hearing. New York: Springer Verlag, 116-154.

Ghiselli A, Laurenti O, De Mattia G, Maiani G, and Ferro-Luzzi A (1992). Salicylate hydroxylation as an early marker of in vivo oxidative stress in diabetic patients. Free Radic Biol Med 13:621-626.

Gonzalez-Vitale JC, Hayes DM, Cvitkovic E, and Sternberg SS (1977). The renal pathology in clinical trials of cisplatinum-diaminedichloride. Cancer 39:1362-1367.

Hannemann J and Baumann K (1988). Cisplatin-induced lipid peroxidation and decrease of gluconeogenesis in rat kidney cortex: Different effects of antioxidants and radical scavengers. Toxicology 51:119-132.

Inoue M, Shimizu C, Shimizu H, and Tanizawa O (1991). Neutralizing effect of sodium thiosulfate on antitumor efficacy of cisplatin for human carcinoma xenografts in nude mice. Gynecol Oncol 40:34-37.

Iwamato Y, Kawano T, Uozumi J, Aoki K, and Baba T (1984). Two-route chemotherapy using high-dose ip cisplatin and iv sodium thiosulfate, its antidote, for peritoneally disseminated cancer in mice. Cancer Treat Rep 68:1367-1373.

Jones MM and Basinger MA (1989). Thiol and thioester suppression of cis-platinum-induced nephrotoxicity in rats bearing the Walker 256 carcinosarcoma. Anticancer Res 9:1937-1942.

Kameyama $Y$ and Gemba M (1991). The iron chelator deferoxamine prevents cisplatin-induced lipid peroxidation in rat kidney cortical slices. Jpn J Pharmacol 57:259-262.

Kamimura T, Whitworth CA, and Rybak LP (1999). Effect of 4-methylthiobenzoic acid on cisplatin-induced ototoxicity in the rat. Hear Res 131:1-2.

Kopke RD, Liu W, Gabaizadeh R, Jacono A, Feghali J, Spray D, Garcia P, Steinman H, Malgrange B, Ruben RJ, Rybak L, and Van De Water TR (1997). Use of organotypic cultures of Corti's organ to study the protective effects of antioxidant molecules on cisplatin induced damage of auditory hair cells. Am J Otol 18:559-571.

Laurell $G$ and Jungnelius U (1990). High-dose cisplatin treatment: Hearing loss and plasma concentration. Laryngoscope 100:724-734.

Lautermann J, Song B, McLaren J, and Schacht J (1995). Diet is a risk factor in cisplatin ototoxicity. Hear Res 88:4753.

Lautermann J, McLaren J, and Schacht J (1995). Glutathione protection against gentamicin ototoxicity depends on nutritional status. Hear Res 86:15-24.
Li G, Frenz DA, Brahmblatt S, Feghali JG, Ruben RJ, Berggren D, Arezzo J, and Van De Water TR (2001). Round window membrane delivery of L-Methionine provides protection from cisplatin ototoxicity without compromising hemotherapeutic efficacy. Neurotoxicology 22:163-176.

Maldonado V, Melendez-Zajgla J, and Ortega A (1997). Modulation of NF- $\kappa$ B, p53 and Bcl-2 in apoptosis induced by cisplatin in HeLa cells. Mutat Res 381:67-75.

Matsushima H, Yonemura K, Ohishi K, and Hishida A (1998). The role of oxygen free radicals in cisplatin-induced acute renal failure in rats. J Lab Clin Med 131:518-526.

Nie Z, Mei Y, Ford M, Rybak L, Marcuzzi A, Ren H, Stiles GL, and Ramkumar $V$ (1998). Oxidative stress increases A1adenosine receptor expression by activating nuclear factor 8B. Mol Pharmacol 53:663-669.

O'Connell MJ and Webster NR (1990). Hyperoxia and salicylate metabolism in rats. J Pharm Pharmacol 42:205-206.

Pomerantz JL and Baltimore D (2000). Comment: Signal transduction. A cellular rescue team. Nature 406:26-27, 29.

Powell SR (1994). Salicylate trapping of $\mathrm{OH}$ as a tool for studying post-ischemic oxidative injury in the isolated rat heart. Free Radic Res 21:355-370.

Ravi R, Somani SM, and Rybak LP (1995a). Mechanism of cisplatin ototoxicity: Antioxidant system. Pharmacol Toxicol 76:386-394.

Ravi R, Somani SM, and Rybak LP (1995b). Pharmacodynamics and biochemical basis for protection of cisplatin otoand nephro-protection by DDTC. Pharmacologist 34:409.

Reser DH, Rho MB, Dewan D, Herbst L, Li G, Stupak HD, Zur K, Romaine J, Frenz D, Goldbloom L, Kopke R, Arezzo J, and Van De Water T (1999). L- and D-methionine provide equivalent long term protection against cisplatin-induced ototoxicity in vivo, with partial in vitro and in vivo retention of antineoplastic activity. Neurotoxicol 20:731-748.

Rybak LP, Ravi R, and Somani SM (1995). Mechanism of protection by diethyldithiocarbamate against cisplatin ototoxicity: Antioxidant system. Fundam Appl Toxicol 26: 293-300.

Rybak LP, Whitworth C, and Somani S (1999). Application of antioxidants and other agents to prevent cisplatin ototoxicity. Laryngoscope 109:1740-1744.

Safinstein R, Winston J, Goldstein M, Moel D, Dikman S, and Guttenplan J (1986). Cisplatin nephrotoxicity. Am J Kidney Dis 8:356-367.

Saito T, Zhang ZJ, Manabe Y, Ohtsubo T, and Saito H (1997). The effect of sodium thiosulfate on ototoxicity and pharmacokinetics after cisplatin treatment in guinea pigs. Eur Arch Otorhinolaryngol 254:281-286.

Schacht J (1999). Antioxidant therapy attenuates aminoglycoside-induced hearing loss. Ann N Y Acad Sci 884:125-130.

Schaumburg HH, Berger AR, and Thomas PK, editors (1992). Anatomical classification of peripheral nervous system disorders. In: Disorders of peripheral nerves, 2 nd ed. Philadelphia: FA Davis Company.

Schweitzer VG (1993). Ototoxicity of chemotherapeutic agents. Otolaryngol Clin North Am 26:759-789.

Sha S-H and Schacht J (1999a). Salicylate attenuates gentamicin-induced ototoxicity. Lab Invest 79:807-813. 
Sha S-H and Schacht J (1999b). Salicylate attenuates cisplatin-induced ototoxicity in the rat (Abstract No. 616). Assoc Res Otolaryngol 22.

Sha S-H and Schacht J (1999c). Formation of reactive oxygen species following bioactivation of gentamicin. Free Radical Biol Med 26:341-347.

Sha SH and Schacht J (2000). Antioxidants attenuate gentamicin-induced free radical formation in vitro and ototoxicity in vivo: D-methionine is a potential protectant. Hear Res 142:34-40.

Stadnick SW, Fleischmann RW, Schaeppi U, and Merriam P (1975). Cis-dichlorodiammineplatinum (II) (NSC-119875): Hearing loss and other toxic effects in rhesus monkeys. Cancer Chemother Rep 59:67-480.

Sugihara KS and Gemba M (1986). Modification of cisplatin otoxicity by antioxidants. Jpn J Pharmacol 40:353-355.

Sugihara KS, Nakano S, Koda M, Tanaka K, Fukuishi N, and Gemba M (1987). Stimulatory effect of cisplatin on production of lipid peroxidation in renal fissues. Jpn J Pharmacol 43:247-252.

Thun MJ, Namboodiri MM, Calle EE, Flanders WD, and Heath CW Jr (1993). Aspirin use and the risk of fatal cancer. Cancer Res 53:1322-1327.
Vollgraf U, Wegner M, and Richter-Landsberg C (1999). Activation of AP-1 and NF- $\kappa$ B transcription factors is involved in hydrogen peroxide-induced apoptotic cell death of oligodendrocytes. J Neurochem 73:2501-2509.

Wang C-Y, Mayo MW, and Baldwin AS (1996). TNF-and cancer therapy-induced apoptosis: Potentiation by inhibition of NF- $\kappa$ B. Science 274:784-789.

Waters GS, Ahmad M, Katsarkas A, Stanimir G, and McKay $J$ (1991). Ototoxicity due to cis-diamminedichloroplatinum in the treatment of ovarian cancer: Influence of dosage and schedule of administration. Ear Hear 12:91-102.

Xu XM, Sansores-Garcia L, Gilroy DW, and Wu KK (1999). Selective suppression of inducible cyclooxygenase 2 gene transcription by aspirin and sodium salicylate. Proc Natl Acad Sci USA 96:5292-5297.

Yin M-J, Yamamoto Y, and Gaynor RB (1998). The antiinflammatory agents aspirin and salicylate inhibit the activity of $I_{\kappa} B$ kinase. Nature 396:77-80.

Zhang JG and Lindup WE (1994). Cisplatin nephrotoxicity: Decreases in mitochondrial protein sulfhydryl concentration and calcium uptake by mitochondria from rat renal cortical slices. Biochem Pharmacol 47:1127-1135.

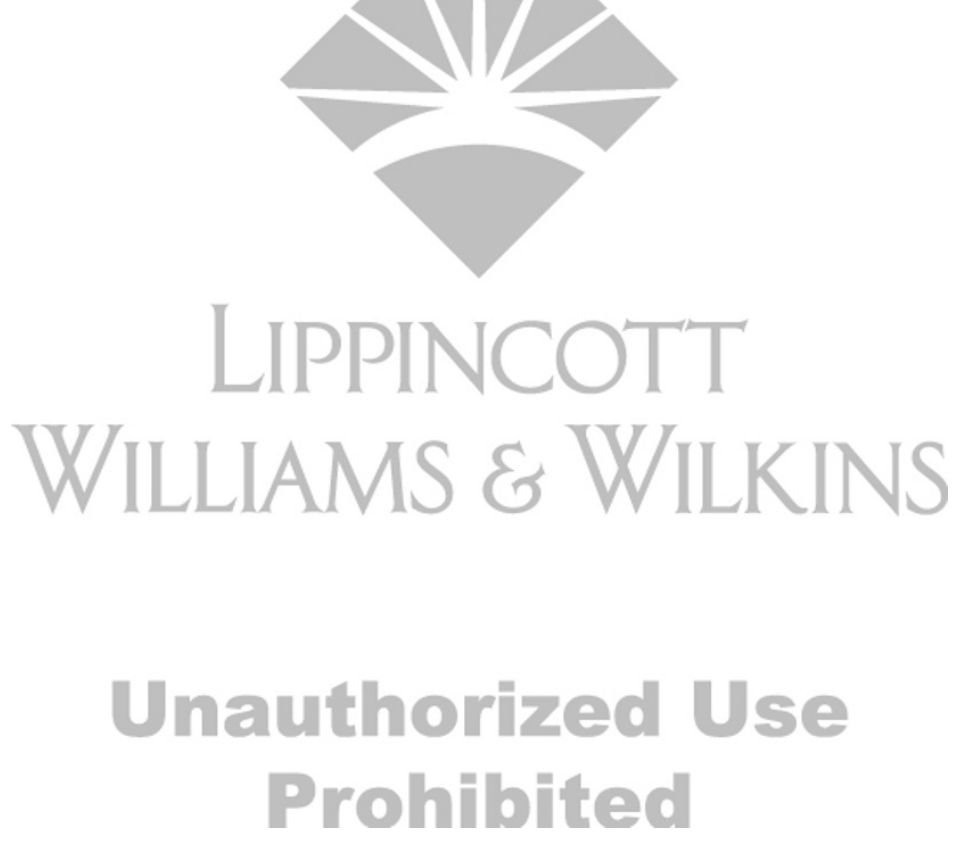

